\title{
Integrated circular optical phased array
}

\author{
Qiankun Liu ${ }^{1, *}$,Tom $\mathrm{Smy}^{1}$, Ahmad Atieh ${ }^{2}$, Pavel Cheben ${ }^{3}$, Alejandro Sánchez-Postigo ${ }^{1,4}$ and Winnie N. Ye ${ }^{1}$ \\ ${ }^{1}$ Department of Electronics, Carleton University, 1125 Colonel By Drive, Ottawa, ON K1S 5B6, Canada \\ ${ }^{2}$ Optiwave Systems Inc, Ottawa, ON, K2E 8A7, Canada \\ ${ }^{3}$ Advanced Electronics and Photonics Research Center, National Research Council Canada, 1200 Montreal Road, Ottawa, ON K1A 0R6, \\ Canada \\ ${ }^{4}$ Currently at Telecommunication Research Institute (TELMA), Universidad de Málaga, CEI Andalucía TECH, Louis Pasteur 35, 29010 \\ Málaga, Spain
}

\begin{abstract}
Existing OPAs are typically based on 2D rectangular arrays or 1D linear arrays. Both approaches present a limited field-of-view (FOV) due to the presence of the grating lobes when the element spacing is larger than $\lambda / 2$. To address the need for an increased steering range, we propose a new design strategy of an OPA system utilizing a 2D circular phased array, with a substantially increased FOV. We present a circular OPA using a demonstrated antenna element design, with an 820 -element array. A steering range $\Omega_{\mathrm{SR}}$ calculated as a solid angle of $0.51 \pi \mathrm{sr}$, and an angular beamwidth of $0.22^{\circ}$, was achieved. The array exhibits a sidelobe suppression larger than $10 \mathrm{~dB}$, and a FOV of $2 \pi \mathrm{sr}$. Although the performance is limited by the far field pattern of the individual antenna we chose, our circular OPA achieved, to the best of our knowledge, the largest steering range reported to date compared to the state-of-the-art integrated optical phased arrays reported in literature.
\end{abstract}

\section{Introduction}

In the last decade extensive research has been reported on static integrated optical phased arrays (OPAs) for solidstate beam steering with fast scanning and high resolution. These OPA systems typically use either a 2D rectangular array of antennas [1, 2], or a 1D linear array of antennas with wavelength sweeping to achieve a two-dimensional beam steering [3-7]. The far field radiation pattern of a rectangular or linear array shows unavoidable strong grating lobes, when element spacing is larger than $\lambda / 2$. Therefore, these arrays demonstrate a restricted aliasingfree beam steering range, exhibiting a limited field-ofview (FOV). To suppress the grating lobes, we propose an OPA system using a 2D circular configuration. By implementing a circular array, the grating lobes are reduced hence increasing the system FOV and the steering range.

The circular OPA provides to date, to the best of our knowledge, the largest steering range compared to other reported integrated OPAs, while keeping the sidelobe suppression larger than $10 \mathrm{~dB}$ [8]. In this work, we present a circular array design: using 820 elements in total to show the superior performance compared to the reported OPAs with a compatible number of elements. As a unique feature of circular arrays, the design shows a theoretical maximum FOV of $2 \pi \mathrm{sr}$, due to the effective suppression of grating lobes.

\section{Beam formation and steering}

\footnotetext{
* Corresponding author: vincentliu3@cunet.carleton.ca
}

A schematic view of our proposed circular array configuration is shown in Fig. 1, the blue dots represent the nano antennas. The circular array contains $\mathrm{N}$ concentric rings whose radius is given by:

$$
R_{n}=R_{0}+(n-1) d r
$$

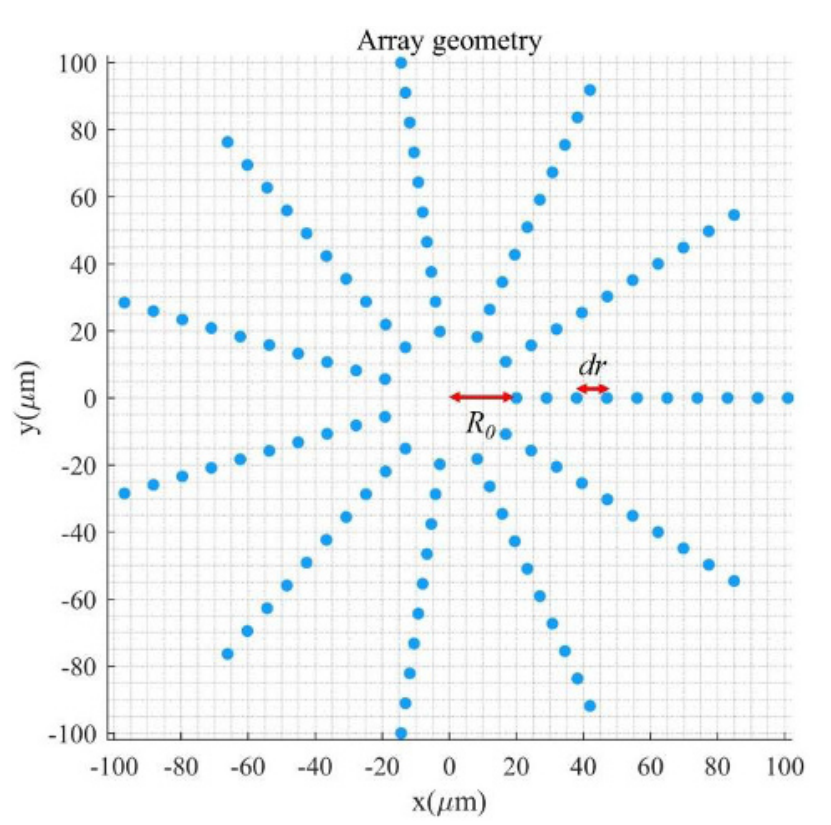

Fig. 1. Schematic view of circular array configuration.

where $R_{0}$ is minimum radius of the first center ring, $\mathrm{n}$ is the number of rings and $d r$ is the delta radius between two adjacent rings. There are $M$ antennas locating in each ring 
with an equal angular spacing between two adjacent antennas in the same ring. In total, $M \times N$ elements are radially arranged in this circular array. Here we assume isotropic antennas, where each identical antenna element emits signal uniformly in all directions. The far-field radiation pattern obtained for the circular antenna array is defined as the array factor, which can be expressed as [9]:

$$
A(\theta, \phi)=\sum_{m=1}^{M} \sum_{n=1}^{N} a_{m n} e^{-i \frac{2 \pi}{\lambda} \sin (\theta) \cos \left(\phi-\frac{2 \pi(m-1)}{M}\right) R_{n}}
$$

where $a_{m n}$ is the element weight coefficient which is equal to $1 /(M \times N)$ for each element, $N$ is the total number of rings, $\lambda$ is the wavelength, $\theta$ and $\phi$ are the elevation and azimuth angles, respectively. As a comparison, in a rectangular array, the array factor is given by Eq. 3 where $\mathrm{M}$ is the number of elements in each row, $\mathrm{N}$ is the number of elements in each column, $\mathrm{dx}$ and dy are the elements spacing in $\mathrm{x}$ and $\mathrm{y}$ axis [9].

$$
\begin{aligned}
& A(\theta, \phi) \\
& =\sum_{m=1}^{M} \sum_{n=1}^{N} a_{m n} e^{-i \frac{2 \pi}{\lambda}\left[d_{x} \sin (\theta) \cos (\phi)+d_{y} \sin (\theta) \sin (\phi)\right]}
\end{aligned}
$$

The array factors are calculated for a wavelength of $1550 \mathrm{~nm}$. Fig. 2a shows the calculated amplitude of array factors, with $N=20, M=41, R_{0}=60 \mu \mathrm{m}, d r=9 \mu \mathrm{m}$, in the $u v$ space where $u=\sin (\theta) \cos (\phi), v=\sin (\theta) \sin (\phi)$. The scanning region corresponds to an entire hemisphere with $1^{\circ}$ resolution to reduce the simulation time $\left(\theta=0^{\circ}: 1^{\circ}: 90^{\circ}\right.$, $\left.\phi=0^{\circ}: 1^{\circ}: 360^{\circ}\right)$. The elevation cut at $\phi=0^{\circ}$, calculated with $0.01^{\circ}$ resolution to measure the angular beamwidth, for the array is shown in Fig. 2b. The inset is the zoom-in view of the array factor when elevation angles are in the range of $-5^{\circ}<\theta<5^{\circ}$. The sidelobe suppression is measured to be $19.16 \mathrm{~dB}$, while the angular beamwidth can be measured as $0.22^{\circ}$. In the applications for autonomous vehicles, it corresponds to a recognition of an object of $38 \mathrm{~cm}$ in size at a distance of $100 \mathrm{~m}$ away from the OPA. In the contrast, considering a same number of elements $N=20, M=41$ and choosing the minimum distance between adjacent elements in the circular array as the element spacing in the rectangular array, i.e., $d_{x}=$ $d_{y}=d r=9 \mu \mathrm{m}$. Fig. $2 \mathrm{c}$ shows the calculated array factor of such rectangular array, a large amount grating lobes can be observed for the rectangular array.

The array far-field pattern (AFP) of the circular array can be calculated as the far-field of each individual antenna $E(\theta, \phi)$ multiplied by the circular array factor $A(\theta, \phi)[1,8]$ :

$$
A F P=E(\theta, \phi) \times A(\theta, \phi)
$$

Using a commercial finite difference time domain (FDTD) tool, by adapting our recent published grating coupler $[11,12]$, we have designed a nano antenna on a silicon-on-insulator waveguide with a $2 \mu \mathrm{m}$ thick upper and buried $\mathrm{SiO} 2$ cladding. It consists of 5 periods, each period contains 4 segments with lengths ( $\mathrm{L} 1=280 \mathrm{~nm}$, L2 $=130 \mathrm{~nm}, \mathrm{~L} 3=196, \mathrm{~L} 4=134 \mathrm{~nm})$. The first segment is subwavelength engineered to have a refractive index of 2.32 in order to reduce the back reflection at the interface of the waveguide and antenna. The grating lines have a circular curvature with a wedge opening angle of $26^{\circ}$. The footprint of this nano antenna is only $5.5 \mu \mathrm{m} \times 2.5 \mu \mathrm{m}$. It shows an upward radiation coefficient of $-1.55 \mathrm{~dB}$ and a back-reflection as low as $-19.94 \mathrm{~dB}$ at $1550 \mathrm{~nm}$. A 3D schematic view of the nano antenna and its far-field radiation pattern are presented in Fig. 3 .
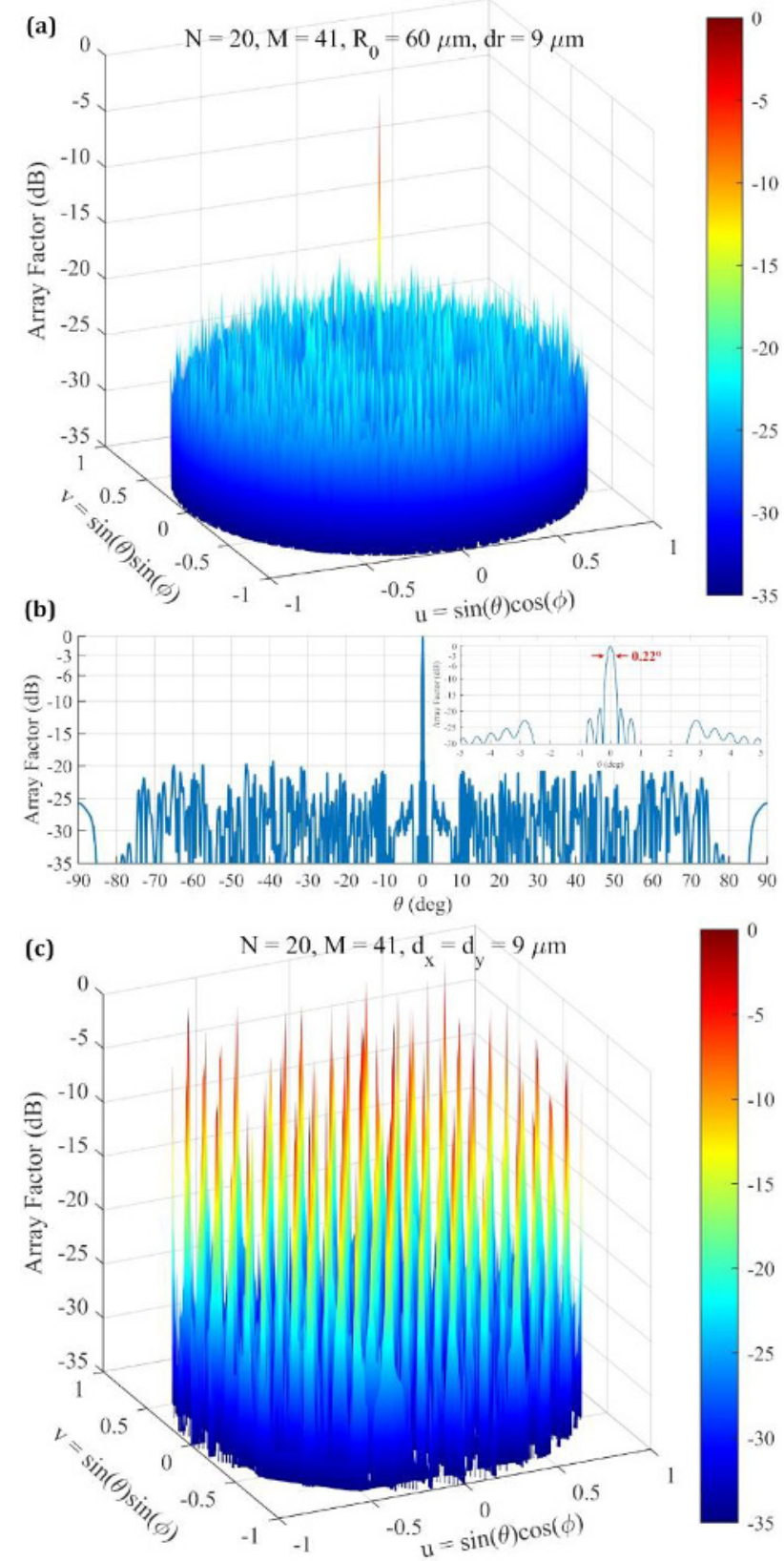

Fig. 2. (a) Simulated amplitude of the array factor in $\mathrm{dB}$ in $u v$ space for Array with $N=20, M=41, R_{0}=60 \mu \mathrm{m}, d r=9 \mu \mathrm{m}$ at $\theta=0^{\circ}, \phi=0^{\circ}$; (b) Elevation cut $\left(\phi=0^{\circ}\right)$ of the array factor of Array 1 ; inset in (b) shows the zoom-in view of the array factor when elevation angles are in the range of $-5^{\circ}<\theta<5^{\circ}$; (c) Simulated array factor in $\mathrm{dB}$ of rectangular array with $N=20, M$ $=41, d_{x}=d_{y}=9 \mu \mathrm{m}$.

Constructing the circular arrays using the above nano antenna, and collaborating with independent controlled phased shifters, we can achieve beam steering over the area within the antenna radiation far field pattern. Fig. 4 
shows the steering range of the array with sidelobe suppression larger than $10 \mathrm{~dB}$. The white circles correspond to the elevation angle $\theta$ from $0^{\circ}$ (surface normal) to $90^{\circ}$, while the straight lines crossed center marked by outer black number represents the azimuthal angle $\phi$ from $0^{\circ}$ to $360^{\circ}$. Because of the irregular shape of the steering range, the steering range is calculated as a solid angle:

$$
\Omega_{S R}=\iint_{\text {steering range }} \sin \theta d \theta d \phi
$$
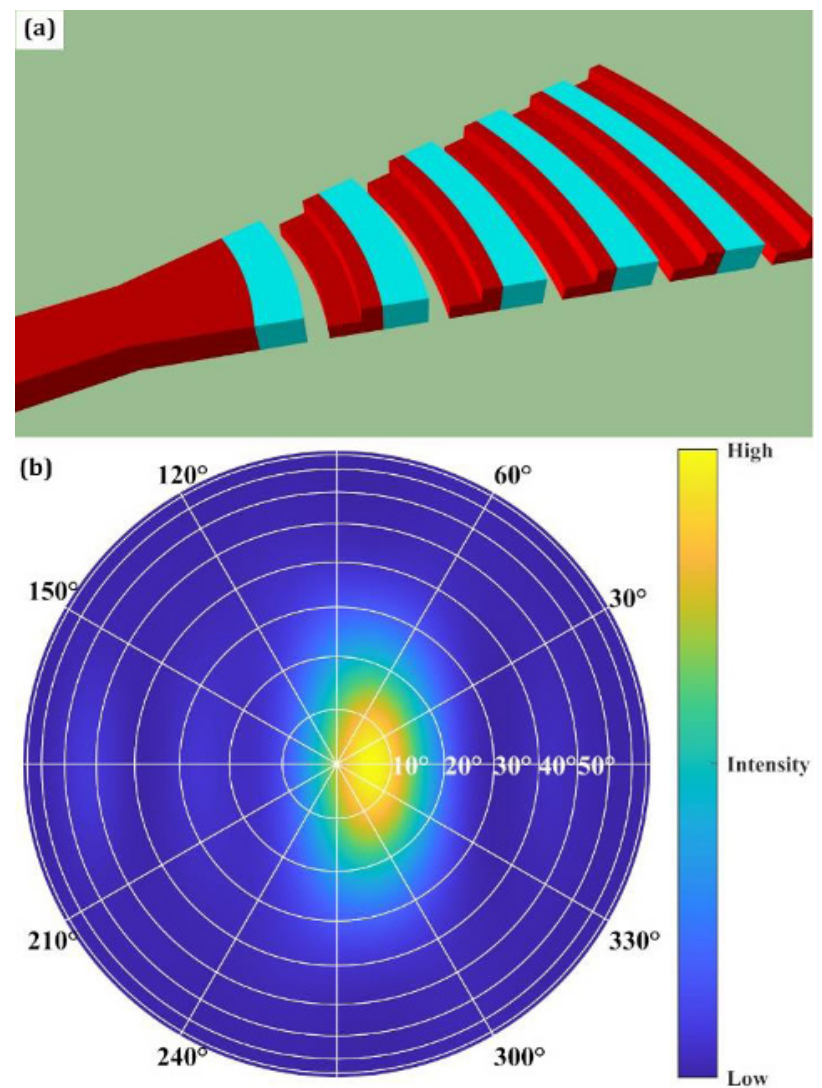

Fig. 3. (a) Schematic 3D view of nano antenna; (b) The corresponding far-field radiation pattern of the optical nanoantenna at $\lambda=1550 \mathrm{~nm}$, calculated from the near-field emission using the near-to-far-field transformation.

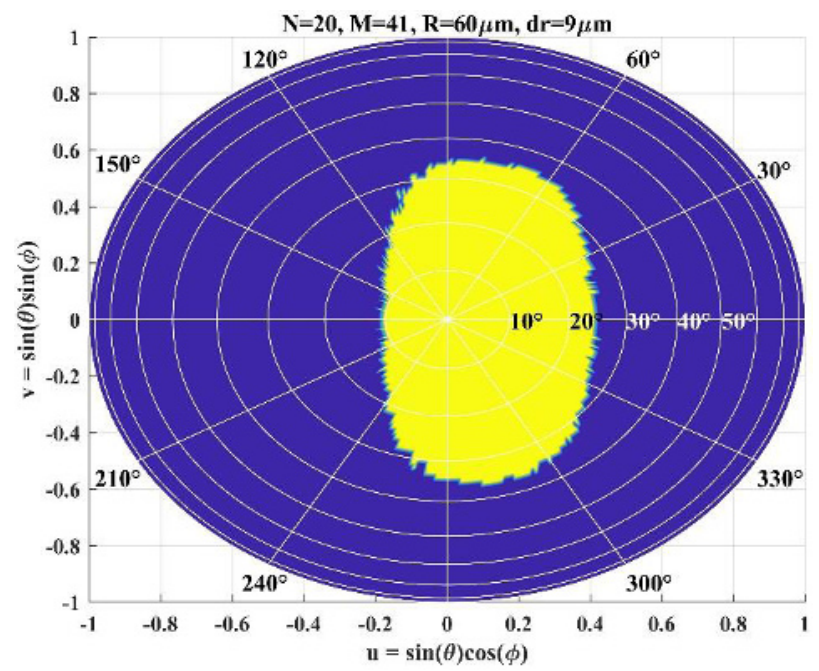

Fig. 4. Steering range of circular array operating at $1550 \mathrm{~nm}$. The yellow area represents the set of all steerable angles where the sidelobe suppression is larger than $10 \mathrm{~dB}$, and the blue area represents non-steerable angles.
According to Eq. 4, we can obtain the steering range of such circular array $\Omega_{\mathrm{SR}}$ of $0.51 \pi$ sr. It covers more than $20 \%$ over the full hemisphere in the forward direction by a single OPA. Moreover, the angular beamwidth of the OPA is as low as $0.22^{\circ}$.

\section{Conclusion}

In this work, we present a new circular optical phased array design which provides an array factor with effectively suppressed grating lobes resulting an overall sidelobe suppression larger than $10 \mathrm{~dB}$. Although theoretically it is possible for the circular OPA to achieve the full FOV of $2 \pi$ sr at a specific wavelength by using ideal isotropic individual nano antenna elements, we use practical pre-designed and demonstrated antenna elements for our numerical studies. With 820 elements in total, the circular OPA provides a $\Omega_{\mathrm{SR}}$ as large as $0.51 \pi \mathrm{sr}$ covering more than $20 \%$ over a hemisphere in the forward direction. We believe that the proposed circular OPA design offers a promising solution to achieve large 2D beam steering range and high angular resolution demanded by applications such as integrated photonic lidars for autonomous vehicles.

\section{References}

1. J. Sun, E. Timurdogan, A. Yaacobi, E.S. Hosseini, and M. R. Watts, Nature 493, (2013)

2. R. Fatemi, A. Khachaturian, and A. Hajimiri, IEEE J. Solid-State Circuits 54, (2019)

3. C.V. Poulton, M. J. Byrd, P. Russo, E. Timurdogan, M. Khandaker, D. Vermeulen, M. R. Watts, IEEE J. Sel. Top. Quantum Electron 25, (2019)

4. S. A. Miller, Y. Chang, C. T. Phare, M. C. Shin, M. Zadka, S. P. Roberts, B. Stern, X. Ji, A. Mohanty, O. A. Jimenez Gordillo, U. D. Dave and M. Lipson, Optica 7, (2020)

5. J. C. Hulme, J. K. Doylend, M. J. R. Heck, J. D. Peters, M. L. Davenport, J. T. Bovington, L. A. Coldren, and J. E. Bowers, Opt. Express 23, (2015)

6. D. N. Hutchison, J. Sun, J. K. Doylend, R. Kumar, J. Heck, W. Kim, C. T. Phare, A. Feshali and H. Rong, Optica 3, (2016)

7. T. Komljenovic, R. Helkey, L. Coldren, and J. E. Bowers, Opt. Express 25, (2015)

8. Q. Liu, T. Smy, A. Atieh, P Cheben and W. N. Ye, IEEE J. Sel. Top. Quantum Electron (submitted)

9. S. J. Orfanidis, Electromagnetic Waves and Antennas, 3rd ed. (2004)

10. M. Kamandar Dezfouli, Y. Grinberg, D. Melati, P. Cheben, J.H. Schmid, A. Sánchez-Postigo, A. Ortega-Moñux, G. Wangüemert-Pérez, R. Cheriton, S. Janz, and D.-X. Xu, Opt. Lett. 45, (2020)

11. S. Khajavi, D. Melati, P. Cheben, J.H. Schmid, Q. Liu, D.X. Xu, and W.N. Ye, Opt. Express 29, 7003 (2021) 